Research Paper

\title{
Pyrroloquinoline Quinone Prevents Estrogen Deficiency-Induced Osteoporosis by Inhibiting Oxidative Stress and Osteocyte Senescence
}

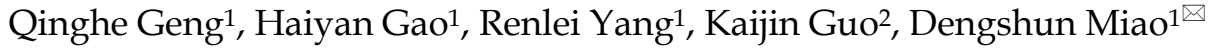 \\ 1. State Key Laboratory of Reproductive Medicine, The Research Center for Bone and Stem Cells, Department of Anatomy, Histology and Embryology, \\ Nanjing Medical University, Nanjing, Jiangsu, China; \\ 2. Department of Orthopedics, The Affiliated Hospital of Xuzhou Medical University, Xuzhou, Jiangsu, China. \\ $\triangle$ Corresponding author: Dr. Dengshun Miao, The State Key Laboratory of Reproductive Medicine, The Research Center for Bone and Stem Cells, Department \\ of Anatomy, Histology, and Embryology, Nanjing Medical University, Nanjing, Jiangsu, 210029, The People's Republic of China. Tel \& FAX: 011-86-25-8686-9377 \\ E-mail: dsmiao@njmu.edu.cn \\ (c) Ivyspring International Publisher. This is an open access article distributed under the terms of the Creative Commons Attribution (CC BY-NC) license \\ (https://creativecommons.org/licenses/by-nc/4.0/). See http://ivyspring.com/terms for full terms and conditions.
}

Received: 2018.02.27; Accepted: 2018.09.08; Published: 2019.01.01

\begin{abstract}
Accumulating studies have shown that oxidative stress increases with aging, which is related to the pathophysiology of postmenopausal osteoporosis. Pyrroloquinoline quinone (PQQ) is a natural anti-oxidant with anti-oxidative and anti-aging effects. However, it is unclear whether PQQ has a protective role against estrogen deficiency-induced osteoporosis. Here, we evaluated the efficacy of $\mathrm{PQQ}$ on bone mineral density, bone microarchitecture, bone turnover and biomechanical strength in ovariectomy (OVX)-induced osteoporosis mouse model. Although dietary PQQ supplement did not affect serum E2 levels and uterine weight in OVX mice, it could prevent OVX-induced bone loss and improve bone strength by inhibiting oxidative stress, osteocyte senescence and senescence-associated secretory phenotype (SASP), subsequently promoting osteoblastic bone formation and inhibiting osteoclastic bone resorption, which was comparable to treatment with exogenous estrogen. The results from our study provide experimental evidence for the clinical use of PQQ to prevent estrogen deficiency-induced osteoporosis.
\end{abstract}

Key words: OVX-induced osteoporosis, PQQ, oxidative stress, osteocyte senescence and SASP.

\section{Introduction}

Osteoporosis is a common systemic disease characterized by low bone mass, poor bone strength and deterioration of bone microstructure [1]. This is due to the uncoupling of bone formation and bone resorption during remodeling [2-4] and could be accelerated in postmenopausal women due to decreased estrogen levels [5]. Estrogen is the regulator of osteoblasts and osteoclasts [6]. In the absence of estrogen, bone resorption is higher than bone formation, resulting in lower bone density and higher fracture rates.

Postmenopausal osteoporosis remains the most common type of osteoporosis, characterized by significant incidence and morbidity of osteoporosis-associated fragility fractures [7, 8].
Although estrogen deficiency is a major cause of osteoporosis, estrogen supplementation is not recommended for its treatment or prevention because of the increased risk of cardiovascular events and cancer of the breast and uterus [7, 9-11]. Parathyroid hormone (PTH), the only clinical agent approved by the US Food and Drug Administration (FDA), could be used to trigger bone formation, but it also increases the bone resorption. It is used for a limit of 2 years for the osteosarcoma potentiality in the rats $[7,9,12]$. Current osteoporosis therapies mainly pay attention to anti-resorption, which may be related to serious side effects, such as the attenuation of bone formation $[7,9]$. Therefore, it is necessary to quest an alternative therapies (natural) with acceptable adverse effects for 
the osteoporosis treatment.

Accumulating studies have exhibited that the reactive oxygen species (ROS)-induced oxidative stress increases with aging, which is related to the pathophysiology of postmenopausal osteoporosis [13-18]. ROS are continuously produced in mitochondria under normal physiological conditions, but an excessive accumulation of ROS will cause damage to the cell membrane, cytoplasm, and ultimately to DNA [19]. The excess of ROS can inhibit osteoblast differentiation and proliferation, enhance osteoclastic differentiation, and ultimately lead to more bone resorption [20, 21]. Dietary supplementation with antioxidants is an effective approach to improve the damage caused by excessive ROS. The stimulatory effects of gonadectomy on oxidative stress, osteoblast apoptosis, and osteoclastogenesis, and the loss of bone mass were reduced by supplementing antioxidants, such as $\mathrm{N}$-acetyl-cysteine (NAC) and ascorbate, which were similar to the treatment of estrogens and androgens $[15,16]$.

Pyrroloquinoline quinone (PQQ) is a redox cycling planar orthoquinone that was originally identified as a coenzyme for methanol dehydrogenase [22]. In the reduced form, the aroxyl radical-scavenging activity of $\mathrm{PQQ}$ is 7.4-fold of vitamin $\mathrm{C}$, indicating that water-soluble PQQ is the most active antioxidant [23]. The previous study suggests that PQQ can enhance mitochondrial functions, induce nerve cells regeneration and maintain neuronal function and fertility [24]. Recently, PQQ has received increasing attention due to its role in free radical scavenging. Previous studies have shown that PQQ played a significantly protective role in the parotid gland, liver, kidney, and bone by inhibiting the oxidative stress and DNA damage from exposure to deleterious events [25-29]. Recent study has demonstrated that PQQ has an inhibitory effect on osteoclast differentiation in vitro [30]. However, the protective effect of PQQ on estrogen deficiency-induced osteoporosis is unclear.

The OVX mouse model is a mature and widely used animal model in the study of postmenopausal osteoporosis [31-33]. We evaluated role and potential mechanisms of PQQ in anti-estrogen deficiency-induced osteoporosis using the OVX mouse model. We assessed alterations in bone tissue changes by measuring bone mass, bone dynamics, bone microarchitecture, bone turnover and bone strength parameters. At the same time, we assessed tissue-level changes in oxidative stress, osteocyte senescence and senescence-associated secretory phenotype (SASP), osteoblast and osteoclast activity. We also treated OVX mice with $17 \beta$-estradiol (E2) to compare its effects with PQQ in estrogen deficiency-induced osteoporosis.

\section{Materials and Methods}

\section{Animals and study design}

The female C57BL/ 6 mice were purchased from the Experimental Animal Center of Nanjing Medical University (Nanjing, China) and used in experiments at 8 weeks of age (25-30 g). All the mice were divided into four groups ( $\mathrm{n}=15$ per group), Three groups were surgically ovariectomized (OVX) via a lateral retroperitoneal approach with ketamine/xylazine anesthesia, while the others were sham-operated (sham control). Paracetamol was used to relieve the pain post-surgery within $24 \mathrm{hr}$.

All the mice except the OVX+PQQ group were fed with a standard AIN-93G diet. The diet for OVX+PQQ mice contained $4 \mathrm{mg} / \mathrm{kg}$ PQQ mixed in the standard mouse chow. OVX mice were injected with $17 \beta$-estradiol (E2) at a dose of $10 \mu \mathrm{g} / \mathrm{kg}$ body weight subcutaneously as a positive control. After 8 weeks, all mice were euthanized by cervical dislocation after anesthesia. Uterus, long bones and blood samples were harvested. The protocol used was approved by the Animal Care Unit and Use Committee of Nanjing Medical University.

\section{Micro-computed tomography}

For micro-computed tomography $(\mu \mathrm{CT})$ analysis, the right femurs were removed and kept in $10 \%$ neutral-buffered formalin. The right femurs were analyzed as described previously [34]. Two-dimensional images were used to generate three-dimensional renderings using the 3D Creator software supplied with the instrument (SkyScan). The following 3D indices were calculated automatically with the software which includes bone mineral density (BMD), bone volume over tissue volume $(\mathrm{BV} / \mathrm{TV})$, trabecular number (Tb.N), trabecular thickness (Tb.Th), trabecular separation (Tb.Sp) and structure model index (SMI, an indicator of the plateor rod-like geometry of trabecular structures) in the secondary spongiosa region; Cortical volume (Ct.V) and cortical thickness (Ct.Th) in the mid-diaphysis.

\section{Biomechanical parameter analysis}

After $\mu \mathrm{CT}$ measurement, right femurs were subjected to the 3-point bending tests as previously described [35]. Biomechanical parameters were calculated as previously described [36].

\section{Histology}

The left femurs were removed from mice and fixed overnight at $4{ }^{\circ} \mathrm{C}$ in $2 \%$ paraformaldehyde which contained 0.075 M-lysine and 0.01 M-sodium 
periodate. These were histology processed as described previously [37].

\section{Histochemical staining for tartrate-resistant acid phosphatase}

Tartrate-resistant acid phosphatase (TRAP) enzyme histochemical analysis was performed on paraffin sections as previously described [38].

\section{Fluorescent measurements}

Mice were injected subcutaneously with calcein $(25 \mathrm{mg} / \mathrm{g}) 10$ and 3 days before death. Femurs were fixed, dehydrated and defatted at $4^{\circ} \mathrm{C}$, and embedded in methyl methacrylate resin. Nonconsecutive $10 \mu \mathrm{m}$ longitudinal sections were cut using a Leica RM2155 (Leica Microsystems, Buffalo Grove, IL, USA), left unstained for dynamic (fluorescent) measurements. Mineral apposition rate (MAR) and surface-based bone formation rate (BFR/BS) were calculated.

\section{Biochemistry assays}

Serum level of 17 $\beta$-estradiol, TRAP-5b, C-terminal cross-linked telopeptides of type I collagen (CTX-I), and serum total antioxidant activities (T-AOC), malonaldehyde (MDA) and superoxide dismutase (SOD) content were measured with respective mice-specific kits (Nanjing Jiancheng Bioengineering Institute, Nanjing, China). All measurements were performed according to the manufacturer's instructions and all samples were assayed in duplicate within the same protocol.

\section{Enzyme-linked immunosorbent assay (ELISA)}

Serum TNF- $\alpha$, IL-1 $\beta$, and IL-6 levels were measured using a standard quantitative sandwich ELISA (MultiSciences Biotech Co., Ltd., Hangzhou, Zhejiang, China).

\section{Flow cytometry analysis for intracellular ROS}

Total long bone marrow cells from different group mice were isolated and the intracellular ROS levels were measured as previously described [39].

\section{Western blot analysis}

For the examination of protein expression level, proteins were extracted from bone tissue, quantitated with a protein assay kit (Bio-Rad, Mississauga, Ontario, Canada). Protein samples $(15 \mu \mathrm{g})$ were fractionated by SDS-PAGE and transferred to nitrocellulose membranes. Membranes were blotted with primary antibodies against SOD1 (Abcam), SOD2 (Novus Biological), Sirt1 (Abcam) and $\beta$-actin (Bioworld Technology, St. Louis Park, MN, USA) were used as loading control. Immunoblotting was carried out as described previously [37].

\section{Immunohistochemistry}

Immunohistochemical staining was carried out as described previously [40] for $\beta$-galactosidase $\left(\beta\right.$-gal), P16 ${ }^{\mathrm{INK} 4 \mathrm{a}}$, using the avidin-biotin-peroxidase complex technique with affinity-purified rabbit anti-mouse $\beta$-gal (Santa Cruz, CA, USA), P16 ${ }^{\text {INK4a }}$ (Santa 192Cruz, CA, USA).

\section{Quantitative real-time RT-PCR}

Total RNA was isolated from mouse bone tissue with Trizol reagent (Invitrogen, Carlsbad, CA, USA) according to the manufacturer's protocol. Real-time RT-PCR was performed as described previously [41] and the primer sequences used for the real-time PCR were purchased commercially. All PCRs were performed in triplicate and the primers used for PCR were shown in Table 1.

Table 1. Sequences of primers employed for RT-PCR. RT-PCR primers used with their name, orientation (S, sense; AS, antisense), sequence, annealing temperature ( $\mathrm{Tm})$, and length of amplicon (bp).

\begin{tabular}{|c|c|c|c|c|}
\hline Name & S/AS & Sequence & $\operatorname{Tm}\left({ }^{\circ} \mathrm{C}\right)$ & $\mathrm{bp}$ \\
\hline \multirow[t]{2}{*}{ RANKL } & Forward & TGG AAG GCT CAT GGT TGG AT & 59 & 75 \\
\hline & Reverse & CAT TGA TGG TGA GGT GTG CA & & \\
\hline \multirow[t]{2}{*}{ OPG } & Forward & TGG AAG GCT CAT GGT TGG AT & 59 & 75 \\
\hline & Reverse & CAT TGA TGG TGA GGT GTG CA & & \\
\hline \multirow[t]{2}{*}{ CTSK } & Forward & CTTCCAATACGTGCAGCAGA & 59 & 155 \\
\hline & Reverse & TCTTCAGGGCTTTCTCGTTC & & \\
\hline \multirow[t]{2}{*}{ TRAP } & Forward & CACTCCCACCCTGAGATTTGT & 60 & 145 \\
\hline & Reverse & CCCCAGAGACATGATGAAGTCA & & \\
\hline \multirow[t]{2}{*}{ IL-1 $\alpha$} & Forward & GTGTTGCTGAAGGAGTTGCCAGAA & 60 & 156 \\
\hline & Reverse & GTGCACCCGACTTTGTTCTTTGGT & & \\
\hline \multirow[t]{2}{*}{ IL-1 $\beta$} & Forward & AAGAGCTTCAGGCAGGCAGTATCA & 60 & 190 \\
\hline & Reverse & TAATGGGAACGTCACACACCAGCA & & \\
\hline \multirow[t]{2}{*}{ IL-6 } & Forward & GGACCAAGACCATCCAAT & 60 & 127 \\
\hline & Reverse & ACCACAGTGAGGAATGTC & & \\
\hline \multirow[t]{2}{*}{ IL-8 } & Forward & ААСТТСТССАСААСССТСТG & 57 & 277 \\
\hline & Reverse & TTGGCAGCCTTCCTGATTTC & & \\
\hline \multirow[t]{2}{*}{ MMP-3 } & Forward & CAAAACATATTTCTTTGTAGAGGACAA & 57 & 91 \\
\hline & Reverse & TTCAGCTATTTGCTTGGGAAA & & \\
\hline \multirow[t]{2}{*}{ MMP-13 } & Forward & ACTTTGTTGCCAATTCCAGG & 57 & 131 \\
\hline & Reverse & TTTGAGAACACGGGGAAGAC & & \\
\hline \multirow[t]{2}{*}{ GAPDH } & Forward & AGA AGG TGG TGA AGC AGG CAT C & 60 & 111 \\
\hline & Reverse & CGA AGG TGG AAG AGT GGG AGT TG & & \\
\hline
\end{tabular}

\section{Computer-assisted image analysis}

The sections from each group were stained with H\&E or histochemically or immunohistochemically and the images of fields were photographed and analyzed using Northern Eclipse image analysis software as described [41, 42].

\section{Statistical Analysis}

Data from image analysis were presented as mean \pm SEM. Two-way ANOVA was used for statistical comparison and to make two-group comparison. After ANOVA Bonferroni test was used with a $\mathrm{P}<0.05$ which was considered significant. 


\section{Results}

\section{Effects of PQQ and estrogen on an OVX-induced bone loss}

To examine whether PQQ supplementation was as effective as exogenous estradiol supplementation in preventing osteoporosis caused by estrogen deficiency, we used OVX mice as an animal model of osteoporosis induced by estrogen deficiency $[43,44]$ After 3 days of OVX, the mice were fed a normal diet with or without PQQ supplementation, or subcutaneously injected with vehicle or $17 \beta$-estradiol (E2) at a dose of $10 \mu \mathrm{g} / \mathrm{kg}$ body weight for 8 weeks, bone phenotype were compared to each other.

We first examined the effects of PQQ or exogenous E2 on serum E2 levels, uterine weight and body weight in OVX mice. Results showed that serum E2 levels and uterine weight were reduced significantly in OVX mice as compared with sham mice, and they did not alter by PQQ supplementation, but both were normalized by exogenous E2 supplementation (Figs. 1A, B). OVX mouse body weight was increased as compared with sham mice, which were normalized by PQQ and exogenous E2 supplementation (Figs. 1C). Then we examined alterations of bone mineral density (BMD) and bone volume parameters using $\mu \mathrm{CT}$ and histomorphometric analyses. BMD, thickness and volume of cortical bone, trabecular number, thickness and bone volume (Figs.1D-J) were all reduced significantly, whereas trabecular separation (Tb.Sp) and structural model index (SMI) was increased markedly in OVX mice compared with sham mice, however, these parameters were normalized by supplementation of both PQQ and E2 (Figs.1K-L). These results demonstrated that dietary PQQ supplementation could prevent estrogen deficiency-induced bone loss as exogenous estrogen supplementation, although serum E2 levels and uterine weight were not affected.
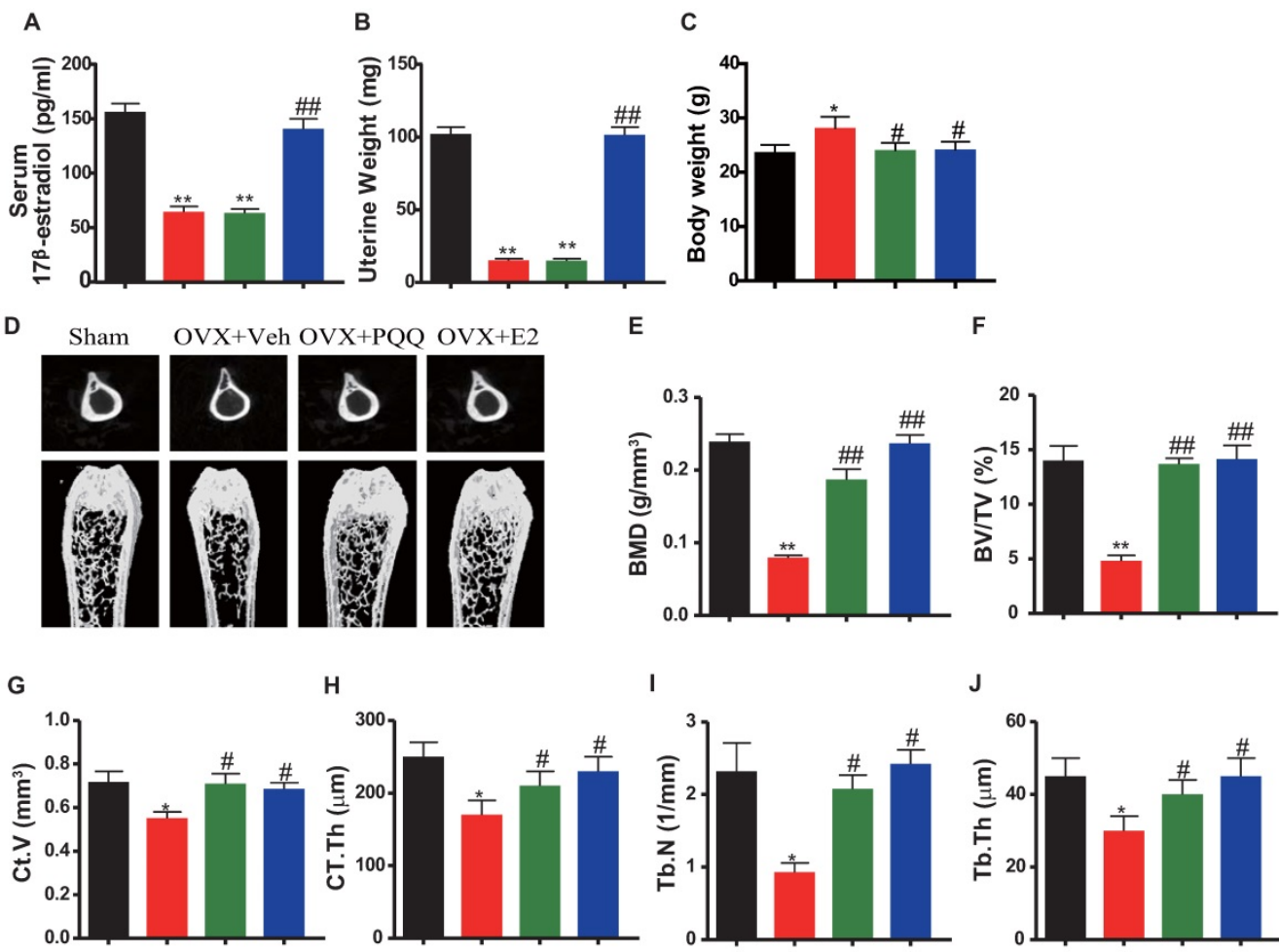

H I

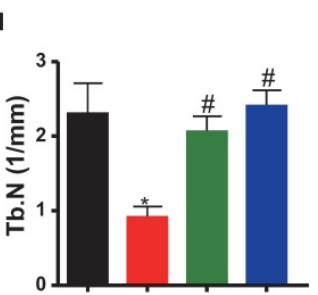

$J$
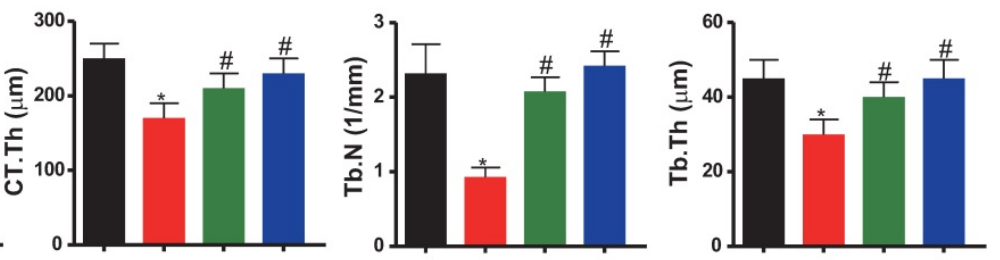

K

L

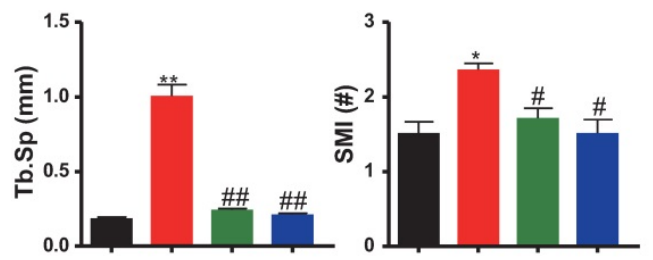

Sham

OVX+Veh

OVX+PQQ

OVX+E2

Figure 1. Effects of PQQ and estrogen on OVX-induced bone loss. (A) Serum 17 $\beta$-estradiol (E2) levels measured by ELISA. (B) Uterine weight. (C) Body weight. (D) The representative micro-CT images of the distal femurs. Analysis of the distal femoral trabecular bone parameters by micro-CT, (E) BMD, (F) BV/TV, (G) Ct.V, (H) CT.Th, (I) Tb.N, (J) Tb.Th, (K) Tb.Sp and (L) SMI. Data represented as mean \pm SEM, $n=15$. N.S. mean no significant. *: $\mathrm{P}<0.05$ and **: $\mathrm{P}<0.01$, vs. the Sham control group. \#: $\mathrm{P}<0.05$ and \#\#: $\mathrm{P}<0.01$, vs. the $\mathrm{OVX}-\mathrm{Veh}$ group. 


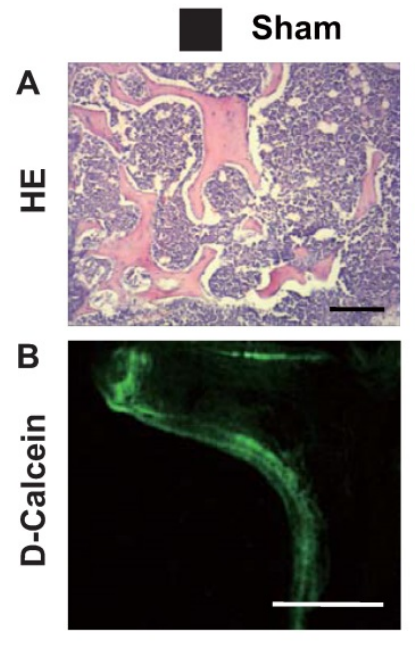

C
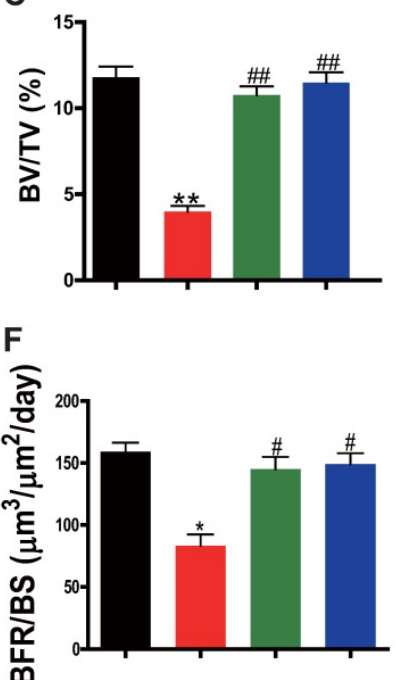
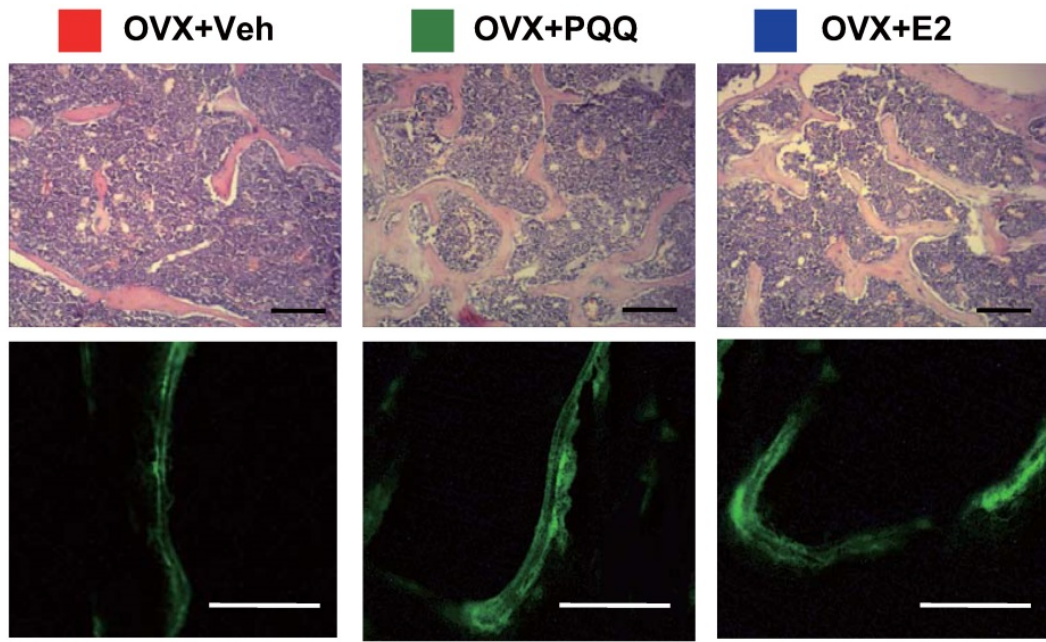

D

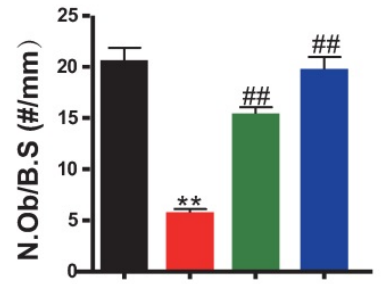

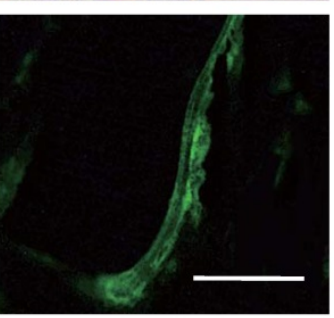

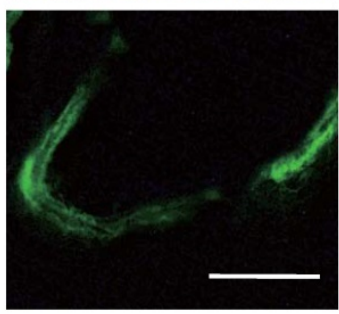

E

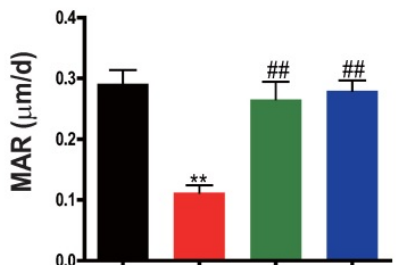

Figure 2. Effects of PQQ and estrogen on osteoblastic bone formation in OVX mice. (A) Representative micrographs of decalcified distal femur paraffin sections stained with H\&E. (B) Representative micrographs of double calcein labeling. Scale bars represent $50 \mu m$ in $A$ and $B$. Histomorphometric analysis for (C) trabecular bone volume/tissue area (BV/TV, \%), (D) N.Ob/B.S (\#/mm), (E) MAR. (F) BFR. Data represented as mean $\pm S E M, n=15$. **: $P<0.01$, vs. the Sham control group. \#: $\mathrm{P}<0.01$, vs. the $\mathrm{OVX}-\mathrm{Veh}$ group.

\section{Effects of PQQ and estrogen on osteoblastic bone formation in OVX mice}

To examine OVX-induced bone loss rescued by PQQ and E2 in association with increased osteoblast bone formation, parameters of osteoblast bone formation were examined by histology, double calcein labeling, and histomorphometric analysis. We observed that there was a significant reduction in osteoblast number, mineral apposition rate (MAR), bone formation rate (BFR/BS) and trabecular bone volume in OVX mice, whereas these parameters were normalized by supplementation of both PQQ and E2 (Figs.2A-F). These results demonstrated that PQQ and E2 could increase osteoblastic bone formation in OVX mice.

\section{Effects of $P Q Q$ and estrogen on the osteoclastic bone resorption in OVX mice}

To examine OVX-induced bone loss rescued by PQQ and E2 was also associated with reduced osteoclast bone resorption, parameters of osteoclast bone resorption were examined by histochemistry, real-time RT-PCR and serum biochemistry. TRAP-positive osteoclast number and surface, RANKL/OPG ratio, gene expression levels of cathepsin $\mathrm{K}$ (CTSK) and TRAP, serum C-terminal telopeptide of type I collagen (CTX-1) and TRAcP5b levels were all increased significantly in OVX mice compared with sham mice, however, these parameters were normalized by supplementation of both PQQ and E2 (Figs.3A-H). These results demonstrated that $\mathrm{PQQ}$ and E2 could inhibit osteoclastic bone resorption in OVX mice. 


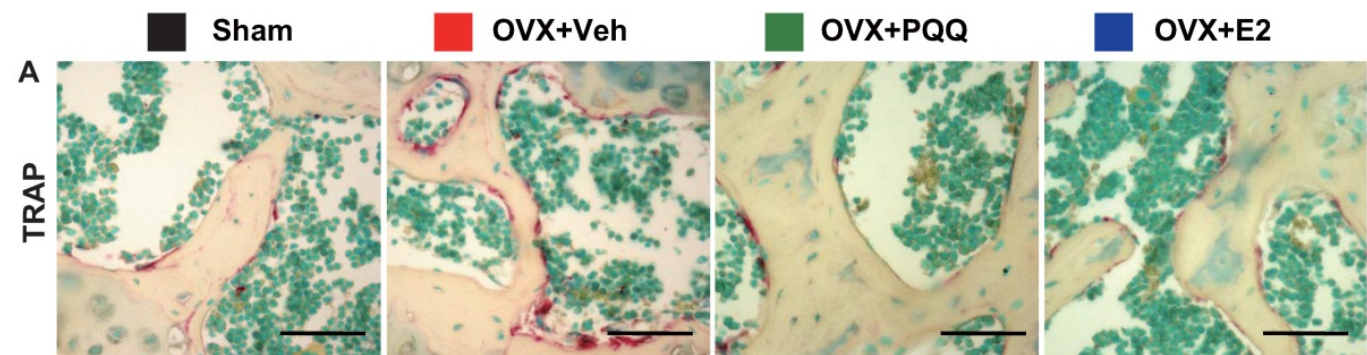

B

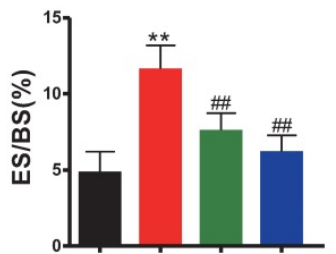

E

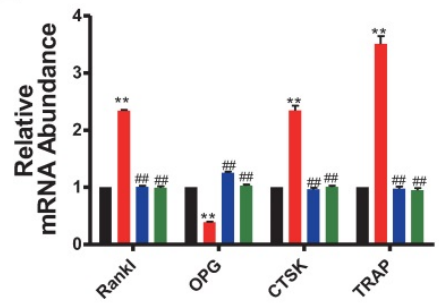

H

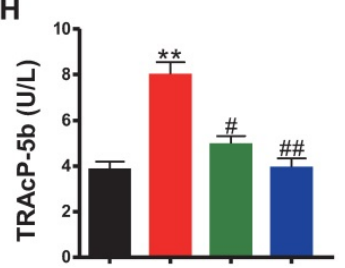

C

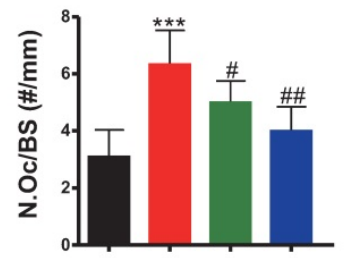

$F$

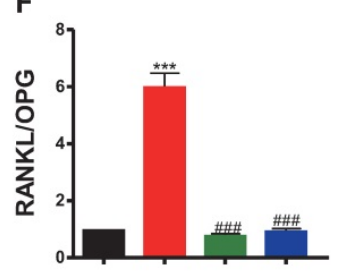

D

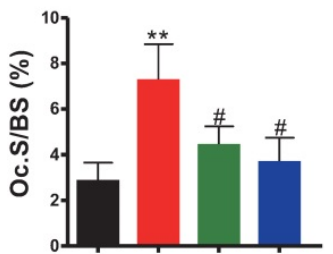

G

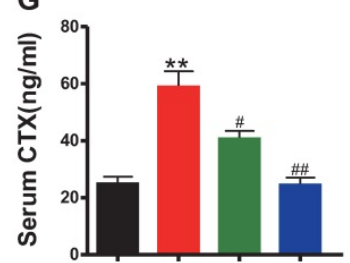

Figure 3. Effects of PQQ and estrogen on the osteoclastic bone resorption in OVX mice. (A) Representative micrographs of distal femur paraffin sections stained histochemically for TRAP. Scale bars represent $50 \mu \mathrm{m}$ in A. For static histomorphometric analysis, (B) eroded surface/bone surface (ES/BS, \%), (C) osteoclast number/bone surface (N.Oc/BS, \#/mm) and (D) Osteoclast surface/bone surface (Oc.S/BS, \%). RT-PCR of bone tissue extracts for (E) the gene expression of RANKL, OPG, CTSK and TRAP and (F) RANKL/OPG ratio. Messenger RNA expression assessed by RT-PCR was calculated as a ratio relative to the GAPDH mRNA level and expressed relative to sham control mice. $(G)$ Serum bone resorption markers CTX-1 and $(H)$ TRAP5b. Data represented as mean \pm SEM, $n=15$. *P $<0.05$ and $* * P<0.01$, vs. the sham control group. \#P<0.05 and \#\#P<0.01, vs. the OVX-Veh group.

\section{Effects of PQQ and estrogen on bone biomechanical indexes in OVX mice}

To determine whether the supplementation of PQQ can improve bone strength in OVX mice as the supplementation of exogenous estradiol, bone biomechanical indexes were examined. Results showed that there was a significant reduction of energy absorption, bending stiffness, maximum load and elastic modulus in OVX mice as compared to sham mice, however, these parameters were normalized by supplementation of both PQQ and E2 (Figs.4A-D). The present results demonstrated that PQQ and E2 also could improve bone strength in OVX mice.

\section{Effects of $P Q Q$ and estrogen on redox balance} in OVX mice

To determine whether the estrogen deficiency-induced osteoporosis prevented by supplementation of PQQ or estrogen is interrelated with reduced oxidative stress induced by OVX, we examined the alterations in parameters of oxidative stress and the antioxidant enzyme activity and expression levels in bony tissue. Results showed that ROS levels and malondialdehyde (MDA) content were increased significantly, whereas total antioxidant capacity (T-AOC) and superoxide dismutase (SOD) activity, protein expression levels of SOD1, SOD2 and Sirt1 were reduced significantly in OVX mice compared with sham mice, however, these parameters were normalized by supplementation of both PQQ and E2 (Figs. 5A-H). These results showed that estrogen deficiency could induce oxidative stress in bony tissue, whereas PQQ or E2 supplementation could reduce oxidative stress in OVX mice. 


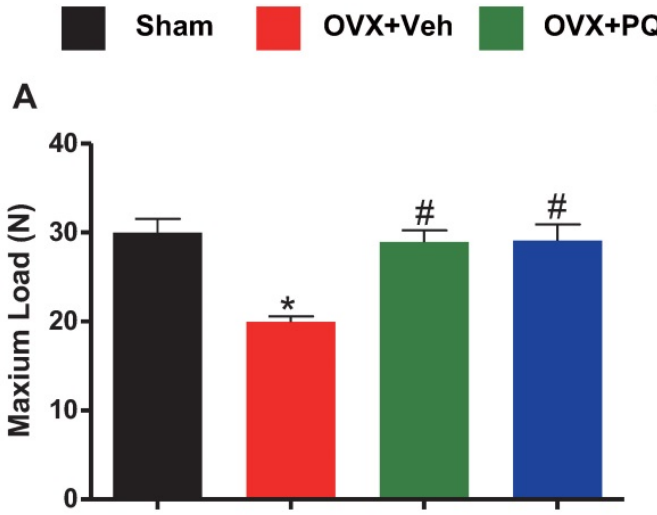

B

C

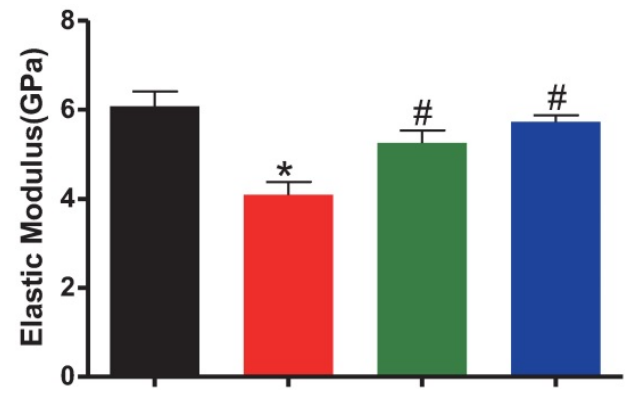

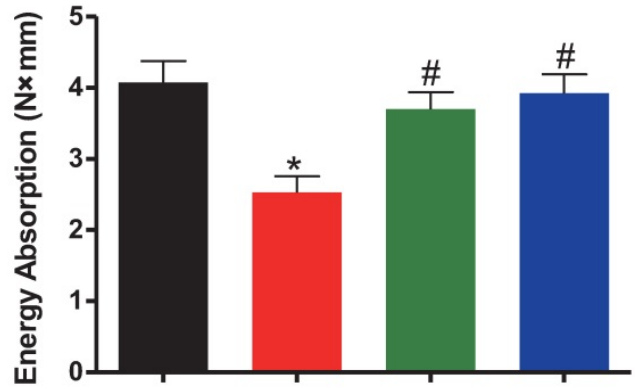

D

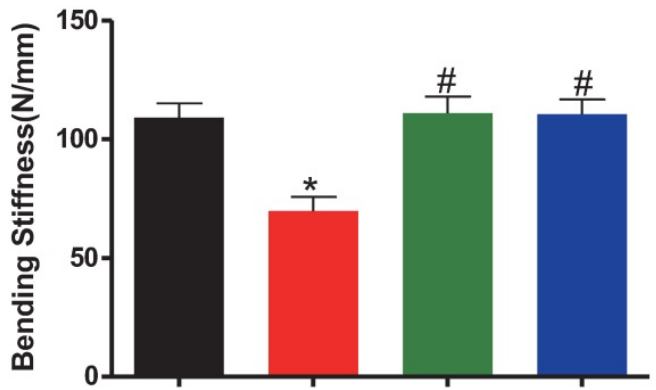

Figure 4. Effects of $\mathbf{P Q Q}$ and estrogen on bone biomechanical indexes in OVX mice. Evaluation of biomechanical properties on femur by using a three-point blending test, including (A) maximum load, $(B)$ energy absorption, $(C)$ elastic modulus and (D) bending stiffness. Data were the means \pm SEM ( $n=15$ for each group). $* \mathrm{P}<0.05$ and $* * \mathrm{P}<0.01$, vs. the sham control group. \#P<0.05 and \#\#<0.01, vs. the OVX-Veh group.
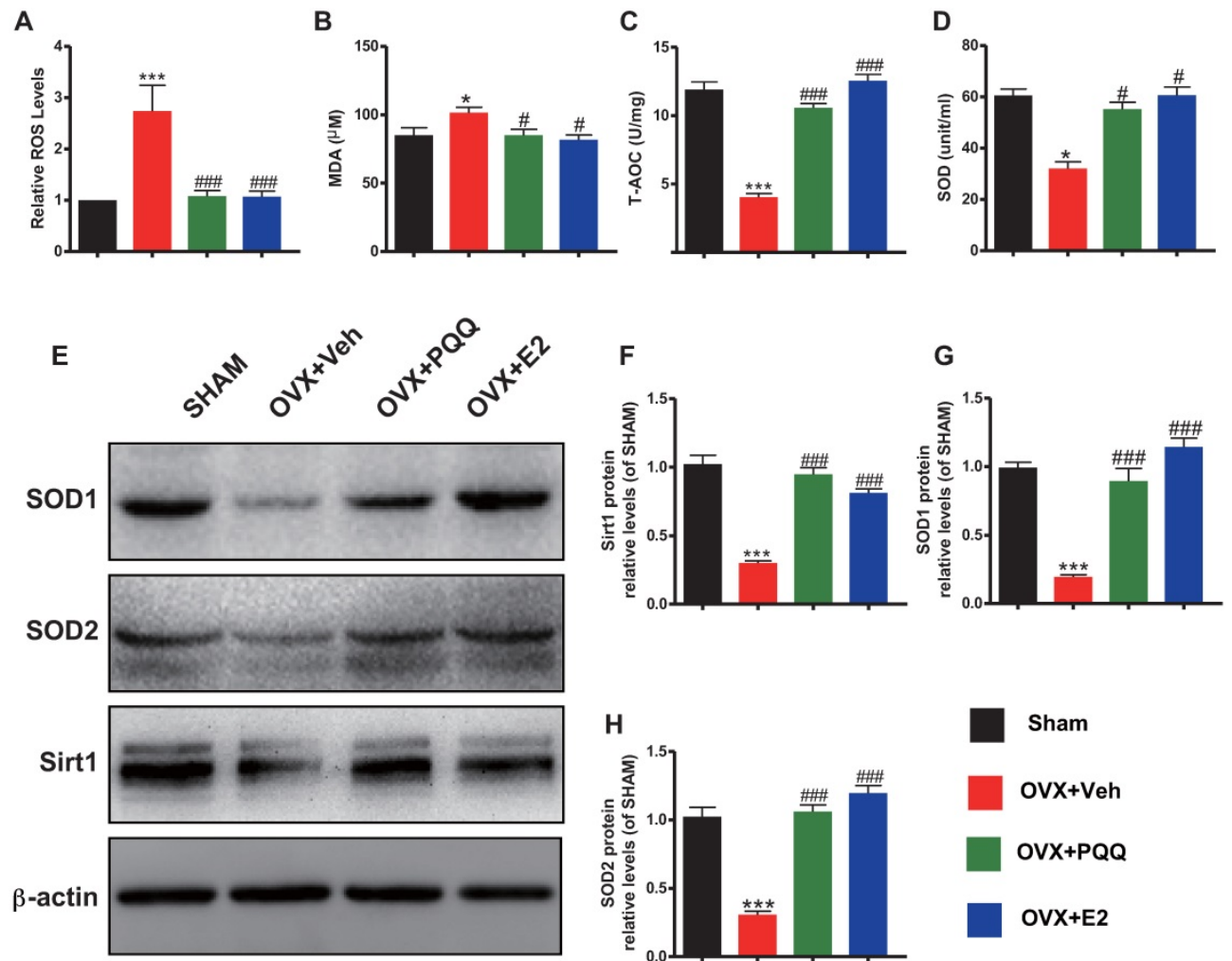

Figure 5. Effects of PQQ and estrogen on redox balance in OVX mice.(A) ROS levels in bone marrow. (B) Serum malonaldehyde (MDA) content, (C) Total antioxidant capacity (T-AOC) and (D) superoxide dismutase (SOD) activity. (E) The protein expression levels of SOD1, SOD2 and Sirt 1 were detected by Western blotting. GAPDH was used as the loading control. (F) SOD1, (G) SOD2 and (H) Sirtl protein levels were assessed by densitometric analysis calculated as a ratio relative to $\beta$-actin protein levels and expressed relative to levels of Sham control mice. Data were the means $\pm S D$ ( $n=6$ for each group). \#P $<0.05$ and \#\#P<0.01, vs. the sham group. $* \mathrm{P}<0.05$ and $* * \mathrm{P}<0.01$, vs. the OVX-Veh group. 

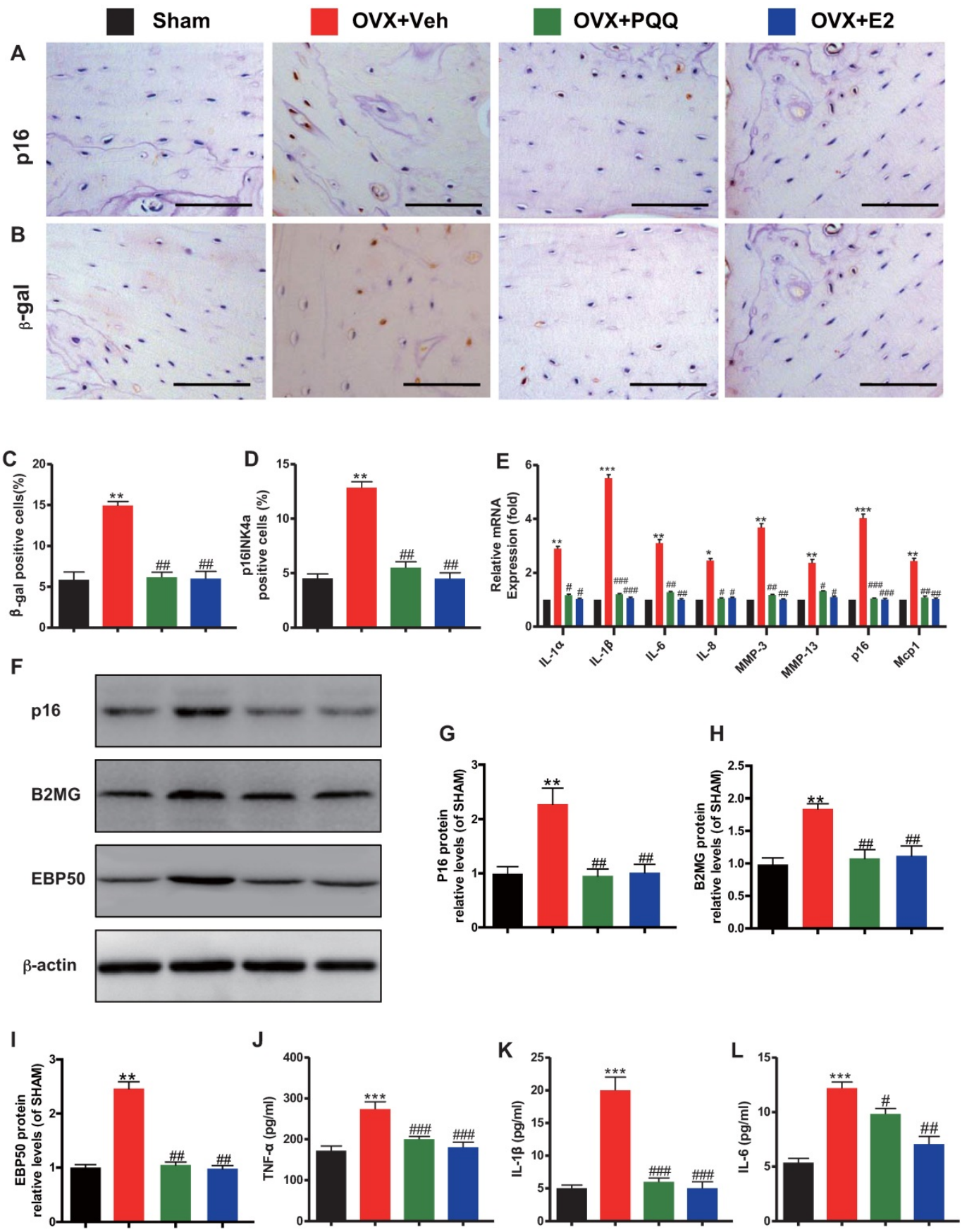

Figure 6. Effects of PQQ and estrogen on osteocyte senescence and SASP in OVX mice. Representative micrographs from paraffin sections of distal femurs with immunohistochemical staining for (A) p16 and (B) $\beta$-gal. Scale bars represent $50 \mu \mathrm{m}$ in A and B. (C) The percentage of p16 positive osteocyte cells and (D) $\beta$-gal positive cells. (E) mRNA expression of established SASP components. Serum pro-inflammatory cytokines (F) TNF- $\alpha$, (G) IL-1 $\beta$, and (H) IL-6 were measured. Data were the means \pm SEM ( $n=6$ for each group). $\# P<0.05$ and \#\#P $<0.01$, vs. the sham group. $* P<0.05$ and $* * P<0.01$, vs. the OVX-Veh group.

\section{Effects of PQQ and estrogen on osteocyte senescence and SASP in OVX mice}

To determine whether the estrogen deficiency-induced osteoporosis prevented by supplementation of PQQ or estrogen is associated with reduced osteocyte senescence and senescence-associated secretory phenotype (SASP) induced by OVX, we examined the alterations of osteocyte senescence and SASP. We found that the percentages of $\mathrm{p} 16$ and $\beta$-galactosidase positive osteocytes (Figs. 6A-D), the gene expression levels of SASP components including IL-1a, IL-1 $\beta$, IL-6, IL-8, Mmp-3 and Mmp-13 (Fig. 6E), the protein expression levels of p16, beta-2 microglobulin (B2MG) and EBP50 (Figs. 6F-I), and serum TNF-a, IL-1 $\beta$ and IL-6 levels (Figs. 6J-L) were all increased significantly in OVX mice compared with sham mice, however, these parameters were normalized by supplementation of both PQQ and E2. These results exhibited that 
estrogen deficiency could induce osteocyte senescence and SASP, whereas PQQ or E2 supplementation could inhibit osteocyte senescence and SASP in OVX mice.

\section{Discussion}

Postmenopausal osteoporosis is the most common form of osteoporosis associated with significant morbidity, mortality, deterioration in the quality of life and financial costs [7]. The OVX mouse model is a mature and widely used animal model in the study of postmenopausal osteoporosis [31-33]. In our current study, we used the OVX mouse model to assess the effects of PQQ on the estrogen-deficiency induced osteoporosis. Firstly, we confirmed that OVX resulted in estrogen deficiency, uterine atrophy and bone loss, whereas exogenous E2 supplementation rescued uterine atrophy and OVX induced bone loss. Simultaneously, we demonstrated that dietary PQQ supplementation did not alter serum E2 levels and uterine weight, but rescued bone loss induced by OVX.

Although the major result of the estrogen deficiency is increased bone resorption, bone formation is maintained by estrogen at the cellular level [43]. Thus we evaluated the effects of OVX, supplementation of E2 or PQQ on bone turnover parameters. Current study demonstrated that OVX not only induced osteoclastic bone resorption but also reduced osteoblastic bone formation and bone strength. In contrast, the bone resorption of osteoclasts and bone formation of osteoblasts and bone biomechanical indices were normalized by the supplementation of E2 or PQQ. Our results show a comparable role of PQQ as estrogen in preventing estrogen deficiency-induced osteoporosis by inhibiting osteoclastic bone resorption, stimulating the osteoblastic bone formation and enhancing bone strength.

Next, we asked whether the estrogen deficiency-induced osteoporosis prevented by supplementation of estrogen is associated with reduced oxidative stress induced by OVX. Recent studies strongly indicate that estrogen deficiency accelerates bone aging and significantly impairs the defense mechanisms of antioxidant stress [14, 44]. Our recent study suggests that estrogen deficiency causes a down-regulation of Bmi1, thus increasing ROS, T cell activation and RANKL production in $\mathrm{T}$ cells, osteoclastogenesis and accelerated bone loss [45]. Our previous studies also showed that estrogen deficiency-induced oxidative stress with reduced antioxidase levels and activity in mouse bone tissue, and impaired osteogenesis and osteoblastic bone formation. The antioxidant NAC administration resulted in intense improvement in osteogenesis and osteoblastic bone formation in OVX mice [16]. Current study confirmed that estrogen deficiency could induce oxidative stress and reduced antioxidase levels and activity, whereas E2 supplement could reduce oxidative stress by increasing antioxidase levels and activity in OVX mice. Results from previous and our recent study, therefore, support that in vivo, estrogen plays a role to stimulate the osteoblastic bone formation and inhibit the osteoclastic bone resorption, at least partially through defense against oxidative stress.

We demonstrated in this study that dietary PQQ supplementation could not alter serum E2 levels and uterine weight, but could rescue bone loss induced by OVX. Thus, we asked whether PQQ plays a comparable role as estrogen in preventing estrogen deficiency-induced osteoporosis through defense against oxidative stress. To answer this question, we treated the OVX mice with PQQ in the supplementary diet from 3 days after OVX for 8 weeks. Our results demonstrated that dietary PQQ supplementation prevented effectively OVX-induced bone loss as it is the replacement with estrogens in mice through played a comparable role as estrogen in defense against oxidative stress. We recent study has suggested that PQQ supplementation can prevent osteoporosis caused by testosterone deficiency [46]. We also demonstrated the anti-osteoporosis role of PQQ by upregulating antioxidant capacity, suppressing oxidative stress and lowering DNA damage, and down-regulating p16, p19, p21, p27, p53 protein expression levels, and inhibiting cell apoptosis [47]. Our results indicate that PQQ not only prevents the estrogen deficiency-induced osteoporosis as effectively as the replacement with estrogens through defense against oxidative stress but also might avoid the side effects caused by estrogen.

ROS has been described as important mediators for cellular senescence progression [48]. Senescent cells can develop SASP consisting of proinflammatory cytokines, chemokines, and extracellular matrix-degrading proteins. The SASP not only has harmful paracrine effects but also has systemic effects [49-51]. The evidence from recent studies suggests that in the development of age-associated disease, cellular senescence is a strong contributor via its growth arrest phenotype and the SASP factor. Thus, we asked whether the estrogen deficiency-induced osteoporosis prevented by supplementation of PQQ or estrogen is associated with reduced osteocyte senescence and SASP induced by OVX. To answer this question, we examined the alterations of osteocyte senescence and SASP. Our results demonstrated that estrogen deficiency could induce osteocyte senescence as shown increased the percentages of p16 and 
$\beta$-galactosidase positive osteocytes, the expression levels of senescence marker proteins including p16, beta-2 microglobulin (B2MG) and EBP50 [52] and SASP, whereas PQQ or E2 supplement could inhibit osteocyte senescence and SASP in OVX mice. Previous research has shown that by using the INK-ATTAC transgenic mouse model, and only $30 \%$ of senescent cells clearance had profound effects on prevention of tissue dysfunction and the onset of multiple age-related pathologies [53]. In our results, it is clear that estrogen deficiency can induce SASP and osteocyte senescence, whereas supplementation of PQQ or estrogen can prevent osteocyte senescence and SASP.

Conclusively, our present study exhibited that estrogen deficiency-induced bone loss was not only related with increased oxidative stress and osteoclastic bone resorption but also related with increased osteocyte senescence and SASP, and reduced osteoblastic bone formation. The dietary PQQ or estrogen supplementation play the same role in preventing osteoporosis induced by estrogen deficiency by inhibiting oxidative stress and osteoclastic bone resorption, preventing osteocyte senescence and SASP, and stimulating osteoblastic bone formation. Therefore, our current research suggests that PQQ could be used for the prevention and treatment of postmenopausal osteoporosis.

\section{Acknowledgments}

This work was supported by grants from the National Natural Science Foundation of China (81471501, 81230009 and 81730066) and from the National Basic Research Program of China (2014CB942900) to D.M.

\section{Competing Interests}

The authors have declared that no competing interest exists.

\section{References}

1. Sambrook P, Cooper C. Osteoporosis. Lancet. 2006; 367: 2010-8.

2. Xu M, Choudhary S, Voznesensky O, Gao Q, Adams D, Diaz-Doran V, et al. Basal bone phenotype and increased anabolic responses to intermittent parathyroid hormone in healthy male COX-2 knockout mice. Bone. 2010; 47: 341-52.

3. Raisz LG. Pathogenesis of osteoporosis: concepts, conflicts, and prospects. Journal of Clinical Investigation. 2005; 115: 3318-25.

4. Raisz LG. Physiology and pathophysiology of bone remodeling. Clinical Chemistry. 1999; 45: 1353-8

5. Rachner TD, Khosla S, Hofbauer LC. Osteoporosis: now and the future. Lancet. 2011; 377: 1276-87.

6. Seeman E, Delmas PD. Mechanisms of disease - Bone quality - The material and structural basis of bone strength and fragility. New England Journal of Medicine. 2006; 354: 2250-61.

7. Kawai M, Moedder UI, Khosla S, Rosen CJ. Emerging therapeutic opportunities for skeletal restoration. Nature Reviews Drug Discovery. 2011; 10: $141-56$.

8. Khosla S, Bellido TM, Drezner MK, Gordon CM, Harris TB, Kiel DP, et al. Forum on Aging and Skeletal Health: Summary of the Proceedings of an ASBMR Workshop. Journal of Bone and Mineral Research. 2011; 26: 2565-78.
9. Reid IR. Short-term and long-term effects of osteoporosis therapies. Nature Reviews Endocrinology. 2015; 11: 418-28.

10. Barrett-Connor E, Grady D, Stefanick ML. The rise and fall of menopausal hormone therapy. Annual Review of Public Health. 2005; 26: 115-40.

11. Nelson ER, Wardell SE, McDonnell DP. The molecular mechanisms underlying the pharmacological actions of estrogens, SERMs and oxysterols: Implications for the treatment and prevention of osteoporosis. Bone. 2013; 53: 42-50.

12. Neer RM, Arnaud CD, Zanchetta JR, Prince R, Gaich GA, Reginster JY, et al. Effect of parathyroid hormone (1-34) on fractures and bone mineral density in postmenopausal women with osteoporosis. New England Journal of Medicine. 2001; 344: 1434-41.

13. Almeida M, Han L, Martin-Millan M, Plotkin LI, Stewart SA, Roberson PK, et al. Skeletal involution by age-associated oxidative stress and its acceleration by loss of sex steroids. Journal of Biological Chemistry. 2007; 282: 27285-97.

14. Manolagas SC. From Estrogen-Centric to Aging and Oxidative Stress: A Revised Perspective of the Pathogenesis of Osteoporosis. Endocrine Reviews. 2010; 31: 266-300.

15. Lean JM, Davies JT, Fuller K, Jagger CJ, Kirstein B, Partington GA, et al. A crucial role for thiol antioxidants in estrogen-deficiency bone loss. Journal of Clinical Investigation. 2003; 112: 915-23.

16. Shi C, Wu J, Yan Q, Wang R, Miao D. Bone marrow ablation demonstrates that estrogen plays an important role in osteogenesis and bone turnover via an antioxidative mechanism. Bone. 2015; 79: 94-104.

17. Garrett IR, Boyce BF, Oreffo RO, Bonewald L, Poser J, Mundy GR. Oxygen-derived free radicals stimulate osteoclastic bone resorption in rodent bone in vitro and in vivo. The Journal of clinical investigation. 1990; 85: 632-9.

18. Lean JM, Davies JT, Fuller K, Jagger CI, Kirstein B, Partington GA, et al. A crucial role for thiol antioxidants in estrogen-deficiency bone loss. The Journal of clinical investigation. 2003; 112: 915-23.

19. Droge W. Free radicals in the physiological control of cell function. Physiological Reviews. 2002; 82: 47-95.

20. Lee NK, Choi YG, Baik JY, Han SY, Jeong DW, Bae YS, et al. A crucial role for reactive oxygen species in RANKL-induced osteoclast differentiation. Blood. 2005; 106: 852-9.

21. Fraser JH, Helfrich MH, Wallace HM, Ralston SH. Hydrogen peroxide, but not superoxide, stimulates bone resorption in mouse calvariae. Bone. 1996; 19: 223-6.

22. Salisbury SA, Forrest HS, Cruse WB, Kennard O. A novel coenzyme from bacterial primary alcohol dehydrogenases. Nature. 1979; 280: 843-4.

23. Akagawa M, Nakano M, Ikemoto K. Recent progress in studies on the health benefits of pyrroloquinoline quinone. Bioscience Biotechnology and Biochemistry. 2016; 80: 13-22.

24. Kumazawa T, Hiwasa T, Takiguchi M, Suzuki O, Sato K. Activation of Ras signaling pathways by pyrroloquinoline quinone in NIH3T3 mouse fibroblasts. International journal of molecular medicine. 2007; 19: 765-70.

25. Huang Y, Chen N, Miao D. Biological effects of pyrroloquinoline quinone on liver damage in Bmi-1 knockout mice. Experimental and therapeutic medicine. 2015; 10: 451-8.

26. Jin J, Lv X, Chen L, Zhang W, Li J, Wang Q, et al. Bmi-1 plays a critical role in protection from renal tubulointerstitial injury by maintaining redox balance. Aging Cell. 2014; 13: 797-809.

27. Wu X, Li J, Zhang H, Wang H, Yin G, Miao D. Pyrroloquinoline quinone prevents testosterone deficiency-induced osteoporosis by stimulating osteoblastic bone formation and inhibiting osteoclastic bone resorption. American Journal of Translational Research. 2017; 9: 1230-42.

28. Huang Y, Chen N, Miao D. Radioprotective effects of pyrroloquinoline quinone on parotid glands in C57BL/6J mice. Experimental and Therapeutic Medicine. 2016; 12: 3685-93.

29. Huang $\mathrm{Y}$, Miao D, Chen N. The treatment effects and mechanisms of pyrroloquinoline quinone on defective teeth and mandible in Bmi-1 knockout mice. Zhonghua kou qiang yi xue za $\mathrm{zhi}=$ Zhonghua kouqiang yixue zazhi $=$ Chinese journal of stomatology. 2015; 50: 496-502.

30. Kong L, Yang C, Yu L, Smith W, Zhu S, Zhu J, et al. Pyrroloquinoline Quinine Inhibits RANKL-Mediated Expression of NFATc1 in Part via Suppression of c-Fos in Mouse Bone Marrow Cells and Inhibits Wear Particle-Induced Osteolysis in Mice. Plos One. 2013; 8.

31. Khosla S, et al. The Unitary Model for Estrogen Deficiency and the Pathogenesis of Osteoporosis: Is a Revision Needed? Journal of Bone and Mineral Research. 2011; 26: 441-51.

32. Bouxsein ML, Myers KS, Shultz KL, Donahue LR, Rosen CJ, Beamer WG. Ovariectomy-induced bone loss varies among inbred strains of mice. Journal of Bone and Mineral Research. 2005; 20: 1085-92.

33. Syed FA, Melim T. Rodent models of aging bone: an update. Current osteoporosis reports. 2011; 9: 219-28.

34. Miao D, Su H, He B, Gao J, Xia Q, Zhu M, et al. Severe Growth Retardation and Early Lethality in Mice Lacking the Nuclear Localization Sequence and C-Terminus of PTH-Related Protein. Proceedings of the National Academy of Sciences of the United States of America. 2008; 105: 20309-14.

35. Styner M, Pagnotti GM, McGrath C, Wu X, Sen B, Uzer G, et al. Exercise Decreases Marrow Adipose Tissue Through ss-Oxidation in Obese Running Mice. Journal of bone and mineral research : the official journal of the American Society for Bone and Mineral Research. 2017; 32: 1692-702.

36. Artsi H, Cohen-Kfir E, Gurt I, Shahar R, Bajayo A, Kalish N, et al. The Sirtuin1 activator SRT3025 down-regulates sclerostin and rescues 
ovariectomy-induced bone loss and biomechanical deterioration in female mice. Endocrinology. 2014; 155: 3508-15.

37. Miao DS, Bai XY, Panda D, McKee MD, Karaplis AC, Goltzman D. Osteomalacia in Hyp mice is associated with abnormal Phex expression and with altered bone matrix protein expression and deposition. Endocrinology. 2001; 142: 926-39.

38. Miao DS, Scutt A. Recruitment, augmentation and apoptosis of rat osteoclasts in $1,25-(\mathrm{OH})(2) \mathrm{D}-3$ response to short-term treatment with 1,25-dihydroxyvitamin D-3 in vivo. BMC musculoskeletal disorders. 2002; 3.

39. Liu J, Cao L, Chen J, Song S, Lee IH, Quijano C, et al. Bmi1 regulates mitochondrial function and the DNA damage response pathway. Nature. 2009; 459: 387-U100.

40. Zhang Y, Chen G, Gu Z, Sun H, Karaplis A, Goltzman D, et al. DNA damage checkpoint pathway modulates the regulation of skeletal growth and osteoblastic bone formation by parathyroid hormone-related peptide. Int J Biol Sci. 2018; 14: 508-17.

41. Xue YB, Zhang ZL, Karaplis AC, Hendy GN, Goltzman D, Miao DS. Exogenous PTH-related protein and PTH improve mineral and skeletal status in 25-hydroxyvitamin D-1 alpha-hydroxylase and PTH double knockout mice. Journal of Bone and Mineral Research. 2005; 20: 1766-77.

42. Miao DS, He B, Jiang YB, Kobayashi $T$, Soroceanu MA, Zhao J, et al Osteoblast-derived PTHrP is a potent endogenous bone anabolic agent that modifies the therapeutic efficacy of administered PTH 1-34. Journal of Clinical Investigation. 2005; 115: 2402-11.

43. Khosla S, Oursler MJ, Monroe DG. Estrogen and the skeleton. Trends in endocrinology and metabolism: TEM. 2012; 23: 576-81.

44. Manolagas SC, Parfitt AM. What old means to bone. Trends in Endocrinology and Metabolism. 2010; 21: 369-74.

45. Li J, Wang Q, Yang R, Zhang J, Li X, Zhou X, et al. BMI-1 Mediates Estrogen-Deficiency-Induced Bone Loss by Inhibiting Reactive Oxygen Species Accumulation and T Cell Activation. Journal of Bone and Mineral Research. 2017; 32: 962-73.

46. Wu X, Li J, Zhang H, Wang H, Yin G, Miao D. Pyrroloquinoline quinone prevents testosterone deficiency-induced osteoporosis by stimulating osteoblastic bone formation and inhibiting osteoclastic bone resorption. Am J Transl Res. 2017 Mar 15; 9(3):1230-1242.

47. Huang Y, Chen N, Miao D. Effect and mechanism of pyrroloquinoline quinone on anti-osteoporosis in Bmi-1 knockout mice-Anti-oxidant effect of pyrroloquinoline quinone. American Journal of Translational Research. 2017; 9: 4361-74.

48. Colavitti R, Finkel T. Reactive oxygen species as mediators of cellular senescence. IUBMB life. 2005; 57: 277-81.

49. Nelson G, Wordsworth J, Wang C, Jurk D, Lawless C, Martin-Ruiz C, et al. A senescent cell bystander effect: senescence-induced senescence. Aging Cell. 2012; 11: 345-9.

50. Coppe JP, Desprez P-Y, Krtolica A, Campisi J. The Senescence-Associated Secretory Phenotype: The Dark Side of Tumor Suppression. Annual Review of Pathology-Mechanisms of Disease. 2010; 5: 99-118.

51. Acosta JC, Banito A, Wuestefeld T, Georgilis A, Janich P, Morton JP, et al. A complex secretory program orchestrated by the inflammasome controls paracrine senescence. Nature Cell Biology. 2013; 15: 978-U221.

52. Althubiti M, Lezina L, Carrera S, Jukes-Jones R, Giblett SM, Antonov A, et al. Characterization of novel markers of senescence and their prognostic potential in cancer. Cell death \& disease. 2014; 5(e):1528.

53. Baker DJ, Wijshake T, Tchkonia T, LeBrasseur NK, Childs BG, van de Sluis B, et al. Clearance of p16(Ink4a)-positive senescent cells delays ageing-associated disorders. Nature. 2011; 479(U): 232-112. 